\title{
Correction to: "Measurement of saturation overshoot under grass cover" by Miloslav Šír et al.
}

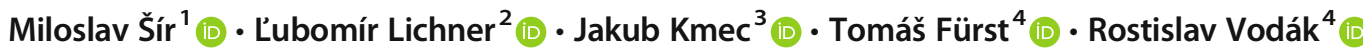

Published online: 28 May 2020

(C) Plant Science and Biodiversity Centre, Slovak Academy of Sciences 2020

Keywords Finger flow · Grass cover · Infiltration experiment $\cdot$ Radioactive tracer technique $\cdot$ Saturation overshoot.

\section{Correction to: Biologia}

https://doi.org/10.2478/s11756-020-00477-z

\section{Methods}

As the activity of iodine 131I decays to half of its initial value in 8.04 days (i.e., in the half-life time), the measured counting rates were corrected for radioactive decay using Eq. (1).

$n=\left(n_{m}-n_{b}\right) \exp (-0.693 t / T)$

where $n$ is the corrected counting rate, $n_{m}$ is the measured counting rate, $n_{b}$ is the counting rate of the natural (background) radioactivity, $t$ is the time elapsed after the start of tracer application, and $T$ is the tracer half-life time.

The online version of the original article can be found at https://doi.org/ 10.2478/s11756-020-00477-Z

Miloslav Šír

milo_sir@yahoo.com

1 Faculty of Fisheries and Protection of Waters, Institute of Aquaculture and Protection of Waters, University of South Bohemia, Na Sádkách 1780, 37005 České Budějovice, Czech Republic

2 Slovak Academy of Sciences, Institute of Hydrology, Dúbravská cesta 9, 84104 Bratislava, Slovakia

3 Joint Laboratory of Optics, Faculty of Science, Palacký University in Olomouc, 17. listopadu 1192/12, 77146 Olomouc, Czech Republic

4 Department of Mathematical Analysis and Applications of Mathematics, Faculty of Science, Palacký University in Olomouc, 17. listopadu 1192/12, 77146 Olomouc, Czech Republic 